\title{
Does Surveillance Increase Survival? Benefits of Periodic Colonoscopy in Patients with Ulcerative Colitis and Colorectal Cancer
}

\author{
Michael J. Stewart ${ }^{1}$
}

Accepted: 29 June 2021 / Published online: 27 July 2021

(c) The Author(s), under exclusive licence to Springer Science+Business Media, LLC, part of Springer Nature 2021

The development of colorectal cancer (CRC) in patients with inflammatory bowel disease (IBD) has long been recognized as a complication of chronic colitis. Nearly a century ago, Dr. Burrell Crohn described carcinomatous degeneration of the bowel as the terminal stage of ulcerative colitis (UC) [1]. While data from mid-twentieth century suggested markedly high rates of CRC, there has been a steady decline in estimated incidence rates in recent decades, with a 2012 metaanalysis of population-based studies estimating 2.4-fold increased risk compared with the general population [2].

Neoplasia associated with IBD differs from sporadic lesions that develop through the well-described adenomato-carcinoma sequence and require special consideration. IBD-associated cancers generally occur a decade earlier and are associated with a poorer prognosis and higher mortality compared with sporadic cancers. They occur within inflamed tissue where distinct microbial and molecular mechanisms may promote neoplasia and are characterized by distinct genomic alterations [3]. The risk of CRC in patients with colonic IBD increases each decade after IBD diagnosis and is generally highest among those with extensive disease, persistent endoscopic inflammation, and a concomitant diagnosis of primary sclerosing cholangitis (PSC).

Periodic colonoscopy has become the accepted surveillance strategy aimed at reducing patient risk through early identification and management of colonic neoplasia. Colonoscopic screening has evolved with the use of high-definition endoscopes and digital imaging techniques or chromoendoscopy that enable improved identification and detection of lesions. Nevertheless, these techniques are not perfect, as demonstrated by a recent retrospective study of 442

Michael J. Stewart

michael.stewart@dal.ca

1 Division of Digestive Care and Endoscopy, Department of Medicine, Dalhousie University, QEII - Victoria Building, 9th floor Victoria Building, 1276 South Park Street, Halifax, NS B3H 2Y9, Canada chromoendoscopic or high-definition colonoscopies during which at least one dysplastic lesion was identified [4]. In this study, $18 \%$ of the detected dysplasia was identified on a random biopsy of normal-appearing mucosa, e.g. not a visible or targeted lesion.

Once dysplasia is identified within the colon of a patient with IBD, the chance of synchronous or metachronous adenocarcinoma increases. The annual risk of progression to CRC among patients previously diagnosed with lowgrade dysplasia (LGD) was estimated to be $0.8 \%$ in a 2017 meta-analysis by Fumery et al. [5]. Due to the significant statistical and clinical heterogeneity, with individual rates ranging from 0.2 to $15.4 \%$, the authors performed a sensitivity analysis excluding low-quality studies and those with a high proportion of patients diagnosed with PSC to produce a more conservative annual risk estimate of $0.4 \%$. Whereas colectomy used to be performed in these patients, current management guidelines support endoscopic resection of lesions where feasible and favour ongoing endoscopic surveillance over colectomy in these cases.

Practice guidelines consider patient factors such as extent of disease, prior LGD, family history, degree of residual inflammation, and the presence of PSC in order to recommend risk-based colonoscopy surveillance intervals of 1-5 years (European guidelines), or 1-3 years (North American guidelines). Though these strategies aim to reduce the incidence and complications of CRC in patients with colonic IBD through early neoplasia detection under the presumption that regular colonoscopy positively impacts overall survival, data supporting a mortality benefit of these surveillance strategies are scarce.

Two case-control studies failed to demonstrate improved CRC-related survival with surveillance colonoscopy, $[6,7]$ whereas a national Dutch cohort study found significantly improved five-year survival amongst patients undergoing surveillance colonoscopy every 1-3 years [8]. A study by Choi et al. [9] identified a cohort of patients with UC who had at least one colonoscopy performed with an 'intent' to 
screen for neoplasia, finding a survival benefit among the patients diagnosed with CRC. A more recent study of those diagnosed with CRC among a cohort of nearly 7000 patients with IBD found that a colonoscopy within the 6-36 months prior to the CRC diagnosis was associated with a decreased incidence of CRC as well as reduced all-cause mortality [10].

In this issue of Digestive Diseases and Sciences, Dr. Neeraj Narula further explored the impact of periodic colonoscopy on the survival of patients with UC following the diagnosis of CRC [11], using an administrative database that houses healthcare utilization information for the entire population of the province of Ontario in order to identify patients with UC. They focussed on those patients that were subsequently diagnosed with CRC during the time period where they would be eligible for colonoscopic surveillance based on current guidelines (i.e. 8 years following the diagnosis of UC).

Of the 62,697 patients with UC and sufficient follow-up information, the authors identified 421 who were diagnosed with CRC during the eligible surveillance period. Unlike prior studies, the majority of these patients (82\%) had undergone at least one follow-up colonoscopy, possibly reflecting changed clinical practice, or perhaps more likely, reflecting the more expansive definition of follow-up procedures used in this study. They included all colonoscopies performed during the eligible surveillance period in order to define the follow-up cohort rather than a more restrictive definition such as that used by Ananthakrishnan et al. [10] where the surveillance cohort was defined as having had a colonoscopy 3-36 months prior to CRC diagnosis.

In the current population-based study by Narula et al. [11], the majority of patients had undergone multiple colonoscopies during the surveillance period with a mean interval of 5.1 years-exceeding all published guidelines and serving as an interesting reflection of real-life practice. They report a $14 \%$ reduction in 15 -year all-cause mortality among patients with UC diagnosed with CRC who had follow-up colonoscopies compared with those who did not $(30.3 \%$ vs. $44.6 \%, p=0.0172$ ). As has been seen in other studies, the progression to CRC in IBD seems to occur at an accelerated rate with a mean time from the last colonoscopy to CRC diagnosis of 3.5 years.

The authors performed logistic regression adjusting for gender, age, urban vs rural location, and the duration of follow-up prior to the diagnosis of CRC. They confirm that all-cause mortality was significantly reduced among patients who underwent follow-up colonoscopy compared with those who do not (OR $0.56,95 \%$ CI $0.32-0.98, p=0.0439$ ). They also identify a small, non-significant, $2.3 \%$ reduction in 15 -year all-cause mortality among those undergoing followup colonoscopy at an interval $<3$ years compared with an interval $>3$ years, which trends towards significance in the adjusted regression model (OR 0.51, 95\% CI 0.26-1.01, $p=0.0523$ ).

While confirming a survival benefit of periodic colonoscopy in patients with long-standing UC, these results diverge somewhat from earlier studies that reported a $41-45 \%$ reduction in 5-year all-cause mortality among UC patients undergoing colonoscopic surveillance $[8,12]$ in that the mortality benefit of periodic colonoscopy reported by Narula et al. [11] is somewhat lower. Although five-year mortality is not assessed, 10-year all-cause mortality did not significantly differ between the study groups $(14.7 \%$ vs. $20.3 \%$, $p=0.2318$ ). Yet, a significant reduction in mortality is seen for 12- and 15-year all-cause mortality with a difference of $17 \%$ and $14 \%$, respectively ( $36.5 \%$ vs. $19.9 \%, p=0.002$ and $44.6 \%$ vs. $30.3 \%, p=0.0172$ ). These data suggest that earlier studies may have overestimated the impact of colonoscopic surveillance on mortality and are more consistent with the $13.8 \%$ mortality benefit reported in a recent meta-analysis evaluating death from CRC [13].

Narula et al. [11] also evaluate the impact of follow-up colonoscopy on the stage of CRC at the time of diagnosis. Rather than evaluate individual cancer stages, the authors grouped cancers more practically into high risk or low risk based on the predicted five-year survival associated with each American Joint Committee on Cancer (AJCC) stage. Patients who underwent more frequent colonoscopy at an interval of $<3$ years were less likely to be diagnosed with a high-risk cancer (41.3\%) compared with those who had colonoscopies at an interval $>3$ years $(48.6 \%)$ and to those who did not undergo follow-up colonoscopy $(77.4 \%, p=0.0048)$.

This study highlights the importance of colonoscopic surveillance programmes for patients with colonic IBD. The use of large administrative databases presents some limitations; however, the study by Narula and colleagues [11] is the first to use population-based data to assess the relationship between periodic colonoscopy and mortality and CRC stage, which may more closely reflect the effect of IBD surveillance in the real-world. Unfortunately, data on established risk factors for IBD-associated CRC and other patient factors were not available, which does limit the generalizability of the findings. Narula et al. [11] confirm that among patients with UC for more than eight years, undergoing follow-up colonoscopy with a mean interval of 5.1 years conveys a mortality benefit compared with no follow-up and suggests that colonoscopy intervals $<3$ years may facilitate CRC diagnosis at an earlier stage.

\section{References}

1. Crohn BB, Rosenberg H. The sigmoidoscopic picture of chronic ulcerative colitis (non-specific). Am J Med Sci 1925;170:220-227. 
2. Jess T, Rungoe C, Peyrin-Biroulet L. Risk of colorectal cancer in patients with ulcerative colitis: a meta-analysis of population-based cohort studies. Clin Gastroenterol Hepatol. 2012;10:639-645.

3. Feagins LA, Souza RF, Spechler SJ. Carcinogenesis in IBD: potential targets for the prevention of colorectal cancer. Nat Rev Gastroenterol Hepatol. 2009;6:297-305.

4. $\mathrm{Hu} \mathrm{AB}$, Burke KE, Kochar B, Ananthakrishnan AN. Yield of random biopsies during colonoscopies in inflammatory bowel disease patients undergoing dysplasia surveillance. Inflamm Bowel Dis. 2021;27:779-786.

5. Fumery M, Dulai PS, Gupta S, Prokop LJ, Ramamoorthy S, Sandborn WJ et al. Incidence, risk factors, and outcomes of colorectal cancer in patients with ulcerative colitis with low-grade dysplasia: a systematic review and meta-analysis. Clin Gastroenterol Hepatol. 2017;15:665-674.e5.

6. Karlén P, Kornfeld D, Broström O, Löfberg R, Persson PG, Ekbom A. Is colonoscopic surveillance reducing colorectal cancer mortality in ulcerative colitis? A population based case control study. Gut. 1998;42:711-714.

7. Lashner BA, Kane SV, Hanauer SB. Colon cancer surveillance in chronic ulcerative colitis: historical cohort study. Am J Gastroenterol. 1990;85:1083-1087.

8. Lutgens M, Oldenburg B, Siersema PD, Van Bodegraven AA, Dijkstra G, Hommes DW et al. Colonoscopic surveillance improves survival after colorectal cancer diagnosis in inflammatory bowel disease. Br J Cancer. 2009;101:1671-1675.
9. Campieri M, Gionchetti P, Belluzzi A, Brignola C, Tampieri M, Iannone $\mathrm{P}$ et al. Topical treatment with 5-aminosalicylic in distal ulcerative colitis by using a new suppository preparation A double-blind placebo controlled trial. Int J Color Dis. 1990;5:79-81.

10. Ananthakrishnan AN, Cagan A, Cai T, Gainer VS, Shaw SY, Churchill $\mathrm{S}$ et al. Colonoscopy is associated with a reduced risk for colon cancer and mortality in patients with inflammatory bowel diseases. Clin Gastroenterol Hepatol. 2014;13:322-329.

11. Narula N, Hu A, Nguyen GC, Rangrej J, Marshall JK. Periodic colonoscopies are associated with improved survival and prognosis of colorectal cancer in ulcerative colitis. Dig Dis Sci. (Epub ahead of print). doi: https://doi.org/10.1007/s10620-021-07151-7.

12. Choi PM, Nugent FW, Schoetz DJ, Silverman ML, Haggitt RC. Colonoscopic surveillance reduces mortality from colorectal cancer in ulcerative colitis. Gastroenterology. 1993;105:418-424.

13. Bye WA, Nguyen TM, Parker CE, Jairath V, East JE. Strategies for Detecting Colon Cancer in Patients with Inflammatory Bowel Disease. Vol. 2017, Cochrane Database of Systematic Reviews. 2017.

Publisher's Note Springer Nature remains neutral with regard to jurisdictional claims in published maps and institutional affiliations. 